\title{
The Effect of 12 Weeks of Training with Total Body Resistance on Static and Dynamic Balance in Older Men
}

\author{
Mohammad Ebrahim Bahram ${ }^{1}$, Roghayyeh Afroundeh ${ }^{2}$, Mohammad Javad Pourvaghar ${ }^{3, *}$ \\ ${ }^{1}$ Ph.D. Student of Sports Physiology, Faculty of Educational Sciences and Psychology, University of Mohaghegh Ardabili, \\ Ardabil, Iran \\ ${ }^{2}$ Assistant Professor of Sports Physiology, Faculty of Educational Sciences and Psychology, University of Mohaghegh Ardabili, \\ Ardabil, Iran \\ ${ }^{3}$ Associate Professor, Department of Physical Education, University of Kashan, Kashan, Iran \\ * Corresponding author: Mohammad Javad Pourvaghar, Department of Physical Education and Sport Science, School of Human \\ Science, University of Kashan, Kashan, Iran. Email: Vaghar@kashanu.ac.ir \\ How to Cite this Article: \\ Bahram ME, Afroundeh R, Pourvaghar MJ. The Effect of 12 Weeks of Training with Total Body Resistance on Static and Dynamic \\ Balance in Older Men. J North Khorasan Univ Med Sci. 2020;6(4):30-38. \\ DOI: $10.29252 / \mathrm{ijrn}-06044$
}

\begin{tabular}{l}
\hline Received: 21 Oct 2019 \\
Accepted: 20 Feb 2020 \\
\hline Keywords: \\
TRX \\
Dynamic Balance \\
Static Balance \\
Elderly \\
\hline C) 2020 Iranian Journal of \\
Rehabilitation Research in Nursing
\end{tabular}

\begin{abstract}
Introduction: Total-Body Resistance Training (TRX) is a new method for increasing muscle strength and decreasing muscle atrophy and improving balance in the elderly. The purpose of this study was to investigate the effect of 12 weeks of TRX training on static and dynamic balance in older men.

Methods: In this quasi-experimental study, 16 older men, with the age range of (62.06 \pm 1.53 years), were selected as sample by availability sampling method; and were randomly placed in two TRX and control groups; each group $n=8$. The experimental group practiced in a TRX exercise program for 12 weeks, each week, three sessions with a duration of 60 minutes. The collected data were analyzed by paired t-test and analysis of covariance (ANCOVA) at a significant level of $\mathrm{P}<0.05$. Results: The results of paired t-test showed that there is significant difference between pre-test and post-test for static $(\mathrm{t}=-22.40, \mathrm{P}=0.0001)$ and dynamic $(\mathrm{t}=13.8, \mathrm{P}=$ $0.0001)$ balance in TRX group. Also the results of ANCOVA test showed that there was significant difference between TRX and control groups in static $(\mathrm{F}=495.45, \mathrm{P}=$ $0.0001)$ and dynamic balance $(\mathrm{F}=74.94, \mathrm{P}=0.0001)$.

Conclusions: It seems that TRX exercises can increase static balance time and decrease dynamic balance time in older men. Probably by using this exercise method, negative physical consequences, and consequently, treatment costs can be reduced. This method can be useful in the field of elderly nursing.
\end{abstract}

\section{Extended Abstract}

\section{OBJECTIVE}

E lderly is a biological process that involves all living things, including humans. This period is the result of the complex interaction of genetic, metabolic, hormonal, immunological, and structural factors that affect body cells, tissues, and systems and their function and cause aging [1]. Muscular strength is one of the active elements in achieving or maintaining balance [5]. On the other hand, the risk of falling increases with decreasing muscular strength in older people. Although nowadays, age-related physical and mental disabilities can be remedied with the use of pharmacotherapy, it seems that more reliable and appropriate approaches must be found to deal with this significant and growing problem of human societies [8, 9]. TRX means total body resistance exercise, and this type of activity enables moving at larger angles as well as more examples of movements than dumbbells or barbells. In this type of action, the injury is much less, and muscles are strengthened by bearing body weight [11]. Aslani et al. (2019) suggested that TRX functional exercises be used to prevention of balance disorder and falling in middle age [14]. Hasanvand et al. (2017) 
reported in a study that performing TRX resistance training has a better effect on improving the lower body balance than traditional resistance training in inactive men [15]. Khajehnemat et al. (2014) contradictory results were reported [20]. Considering that there is a possibility of muscle and joint damage for the elderly due to the use of dumbbells and weights, the issue of balance and falling is one of the critical issues related to the field of elderly health and conflict results have been reported in previous studies, therefore the present study aimed to answer this question that whether 12 weeks of TRX exercise affect dynamic and static balance in elderly males? And that if this method of weight training is useful in developing muscle strength and improving the balance of the elderly?

\section{MATERIALS AND METHODS}

The statistical population of the present study was retired teachers of physical education in Kashan city that, after the announcement, 32 of them agreed to cooperate in the research. The statistical sample of this study was 16 older people who had the conditions to enter the survey. In this study, standing on one leg (Flamingo Balance Test) and round trip test (Timed Up and test) were used for measuring static and dynamic balance, respectively. The experimental group performed Pilates exercises for 12 weeks, three sessions per week, each session 60 minutes. Exercise intensity was also controlled by the Borg pressure perception scale (RPE).

\section{Data Analyzing}

In statistical analysis, paired t-test and covariance analysis (ANCOVA) were used to measure intra-group differences and between groups' differences. Significant level was considered $\mathrm{P}<0.05$.

\section{RESULTS}

Results of paired t-test showed that differences between pre-test and post-test for static balance $(\mathrm{t}=-22.40, \mathrm{P}=$ $0.0001)$ and dynamic balance $(\mathrm{t}=13.8, \mathrm{P}=0.0001)$ were significant in experimental group. Results of covariance analysis test revealed that there were also significant differences between experimental and control groups for static balance $(\mathrm{F}=495.45, \mathrm{P}=$ $0.0001)$ and dynamic balance $(\mathrm{F}=74.94, \mathrm{P}=0.0001)$.

\section{CONCLUSION}

As the body needs to maintain its stability at unstable levels during TRX exercises, this helps to the coordination of the nervous system and improves joint movement. It also increases the strength of joints because, during these exercises, the person moves at a constant speed in one direction [23]. Improving static and dynamic balance as a result of TRX exercises can be due to the facilitation of involvement of fast-twitch and large motor units, increasing in the coordination of muscles, applying stress on the nervous and muscular systems, and the process of removing of autoinhibition and psychological factors. Also, improvement in the motion range in joints and nervous control of movements can be accounted for the benefits of TRX exercises [25]. However, Henwood (2006), in a study titled "Impact of Diverse Training Program (Resistance Training) on Improving the strength and Performance of the Elderly," failed to confirm the effect of physical activity on dynamic and static balance in the elderly [18]. The reason for this discrepancy can be attributed to the duration and nature of the training program used in Henwood's and the present study.

On the other hand, it can be argued that improvement in balance in the elderly is more due to neuromuscular coordination than muscular hypertrophy $[29,30]$. The results of the present study show that TRX exercises as a useful tool can help older people to improve their static and dynamic balance. Therefore, it seems that by using this type of training, the negative physical and psychological consequences and subsequent therapeutic costs can be reduced. TRX exercises may help the successful aging process and be used as an effective method in the field of nursing and elderly rehabilitation.

\section{Ethical Considerations}

This study obtained its ethical approval from the Research Ethics Committee of Kashan University of Medical Sciences (Code: IR.KUAC.REC.75286) and is registered in the clinical trial (IRCT2017082712796N3). All participants of the study signed a written consent form and were assured of the confidentiality of their information.

\section{Funding}

Part of the present study was conducted with the financial support of the Honorable Research ViceChancellor of Mohaghegh Ardabili University and the Honorable Research Vice-Chancellor of Kashan University.

\section{Authors' Contributions}

Mr. Mohammad Ebrahim Bahram is in charge of the original writing and preparation of the initial draft of the article, the main idea, and the laboratory work. Dr. Roghayeh Afroundeh did the initial purpose of studying and analyzing the statistics and part of the writing of the article. Dr. Mohammad Javad Pourvaghar did the initial idea of studying and preparing the draft.

\section{Conflicts of Interest}

The authors declare no conflict of interest

\section{Applicable Remark}

This study is useful for improving balance in the elderly and their quality of life and mental health.

\section{Acknowledgments}

The researchers of this study sincerely thank all the participants who participated in this study. We want to thank the esteemed Vice Chancellor for Research of Kashan University and Mohaghegh Ardabili University, who accompanied us in this research. 


\title{
اثر دوازده هفته تمرينات با مقاومت كل بدن بر تعادل ايستا و يويا در مردان سالمند
}

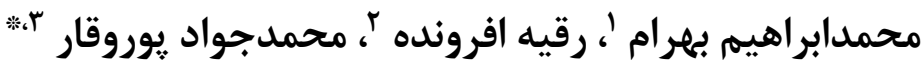

دانشجوى دكترى فيزيولوزى ورزشى، كروه تربيت بدنى و علوم ورزشى، دانشكده علوم تربيتى و روان شناسى، دانشكاه محقّق اردبيلى،

اردبيل، ايران

r استاديار فيزيولوزى ورزشى، كروه تربيت بدنى و علوم ورزشى، دانشكده علوم تربيتى و روان شناسى، دانشكاه محقّق اردبيلى، اردبيل، ايران

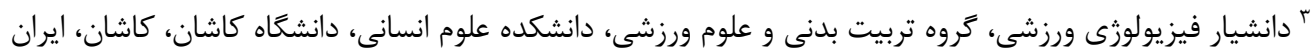

* نويسنده مسئول: محمدجواد يوروقار، دانشيار فيزيولوزى ورزشى، گروه تربيت بدنى و علوم ورزشى، دانشكده علوم انسانى،

دانشخاه كاشان، كاشان، ايران. ايميل: Vaghar@kashanu.ac.ir

تاريخ يذيرش مقاله: • آ/ ||

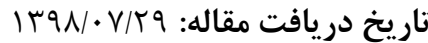

جكيده

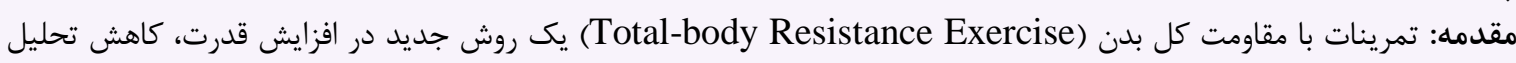

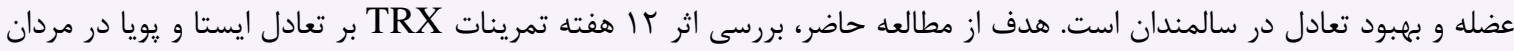
سالمند بود.

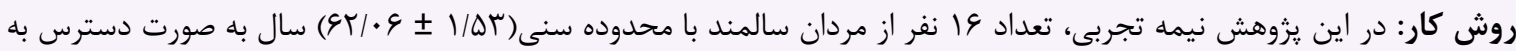

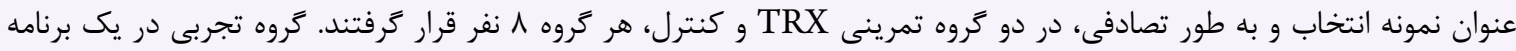

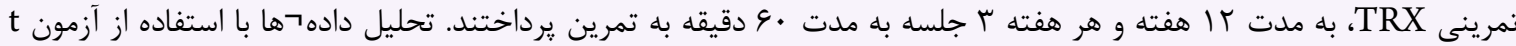

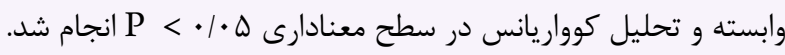

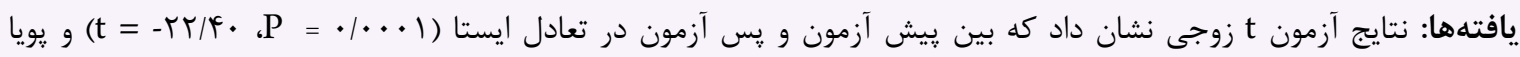

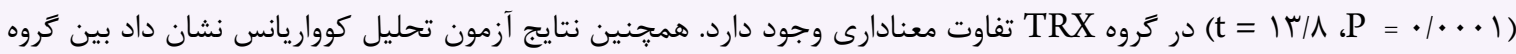
و كنترل در تعادل ايستا ( TRX

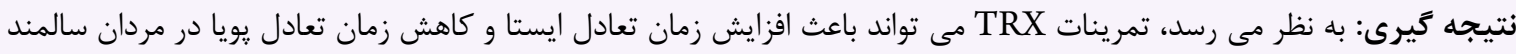

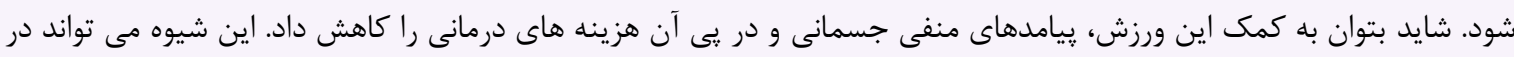
حوزه يرستارى سالمندى مفيد واقع شود. كليدوازهها: TRX، تعادل يويا، تعادل ايستا، سالمند نوديد

تمامى حقوق نشر براى انجمن علمى يرستارى ايران محفوظ است.

مقدمه

شامل تعامل پِيجيده بين عوامل محيطى، بينايى و عوامل عضلانى و همجنين اثر متقابل بين شبكه هاى عصبى و محصول حركتى است كه

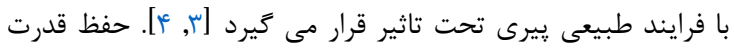
عضلانى يكى از عوامل موثر در احراز يا ميزان موفقيت در حفظ تعادل

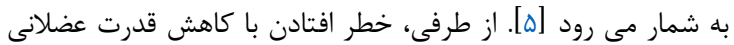
در سالمندان افزايش مى يابد. عواقب شديد افتادن در سالمندان شامل
سالمندى، يك فرايند زيستى است كه تمام موجودات زنده از جمله

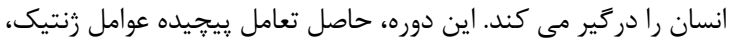

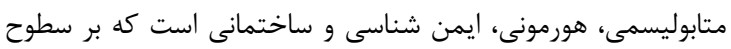

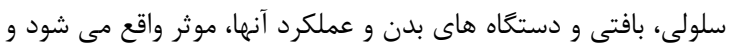

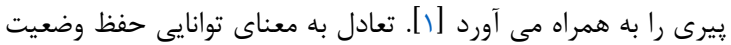

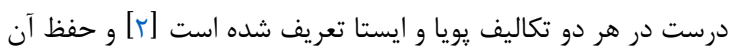




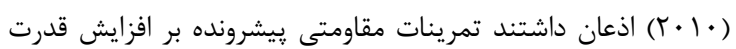

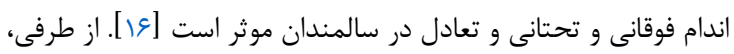

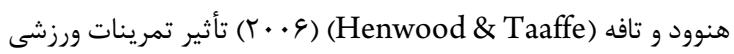

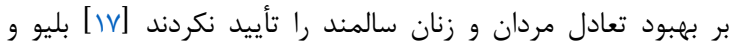

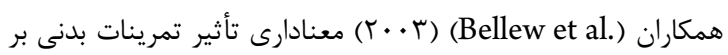
تعادل ايستا و يويا در مردان و زنان ميانسال و سالمند را تأييد نكردند

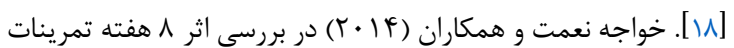
قدرتى بر تعادل ايستا و يويا در مردان سالمند نشان دادند، تمرينات

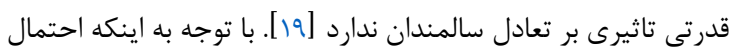

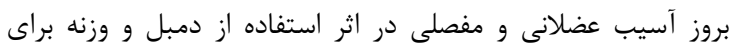

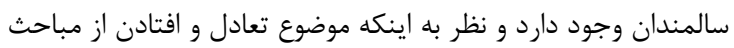
كليدى مرتبط با حوزه سلامت سالمندى است و نتايج حاصل از مطالعات، يافته هاى متناقضى را ززارش كرده اند و از طرفى، در حال

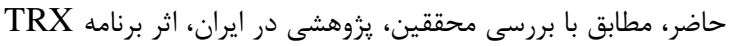

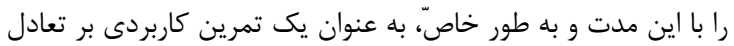

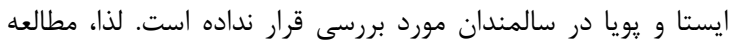
حاضر در يى ياسخ به اين سوال است كه آيا با هفته تمرينات TRX

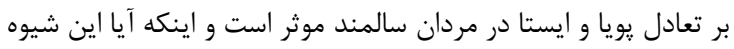
تمرينات با بهره كيرى از وزن بدن مى تواند در توسعه قدرت عضلانى مردي

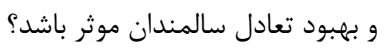

\section{روش كار}

اين يزوهش از نوع نيمه تجربى و كاربردى و طرح آن به صورت يِ

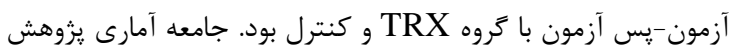
حاضر دبيران مرد بازنشسته تربيت بدنى شهرستان كاشان بودند كه بعد أردان

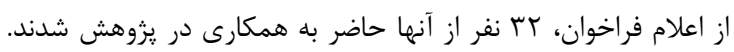

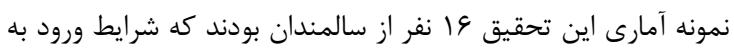

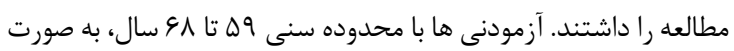
هدفمند و در دسترس انتخاب و بر اساس شاخص توده بدن، همخن

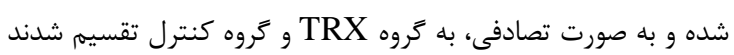

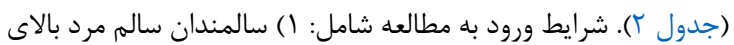

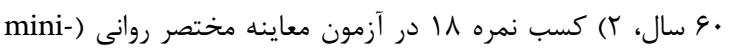
mental state examination (MMSE

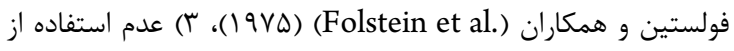

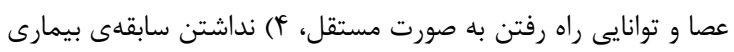

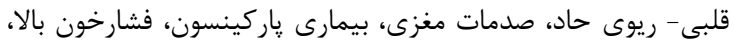

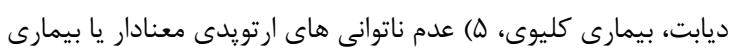

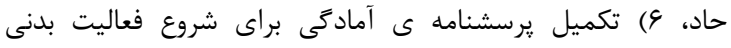
و physical activity readiness questionnaire(PARQ) پيشينه ى يزشكى و V آشنايى آزمودنى ها با نحوه انجام فعاليت بدنى

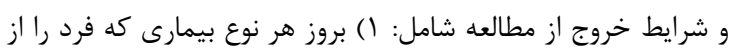

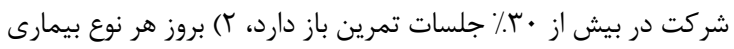

ضربه هاى مغزى و شكستخى هاى استخوان ران است، كه هزينه هاى

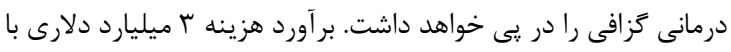

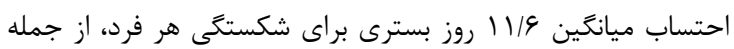

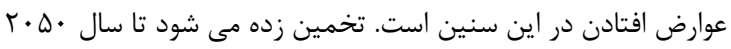

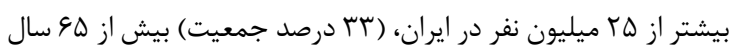

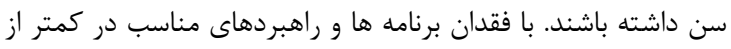

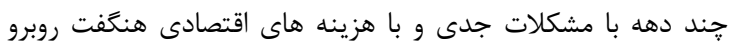

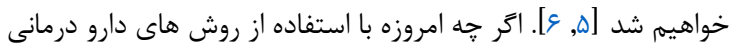

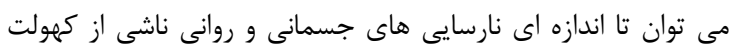
سن را برطرف كرد، اما به نظر مى رسد براى مقابله با اين معضل بزرى

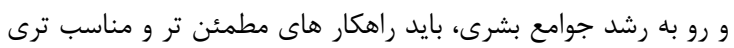

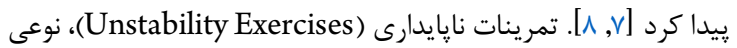

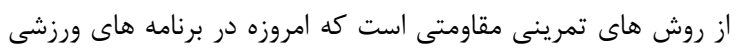

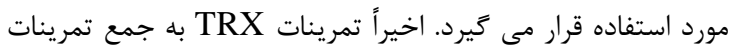

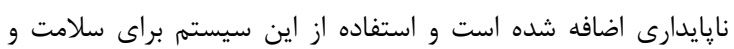

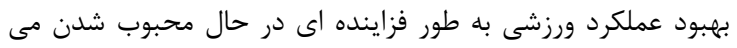

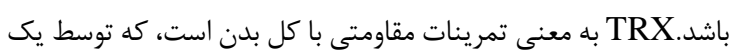

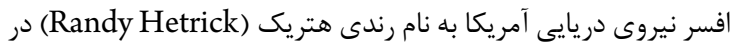

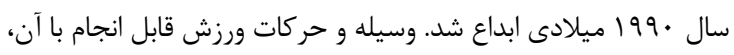

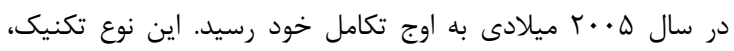
تمرينات مختلف را توسط دو منبع هميشه در دسترس، يعنى جاذبه و

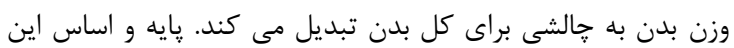

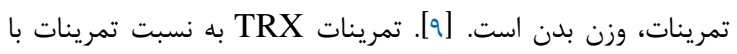

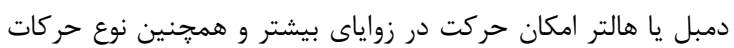
بيشترى را ممكن مى سازد. در اين نوع تمرينات صدمات بسيار كمتر

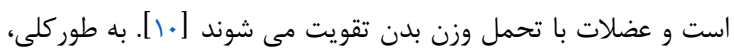

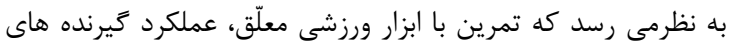

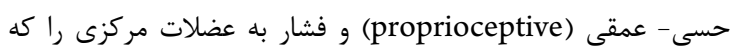

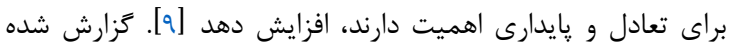
است، روش تمرينى TRX براى تمامى گروه هاى سنى بزرگسال در داري

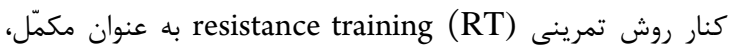

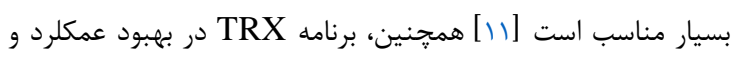
انجام فعاليت هاى روزمره زندكى در سالمندان و افزايش تواناى هاى

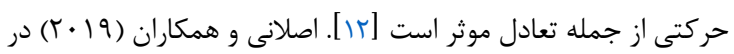

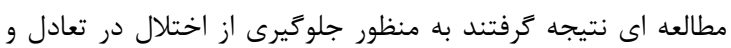

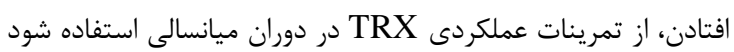

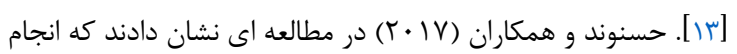

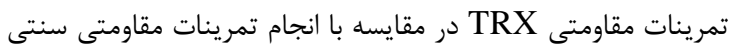

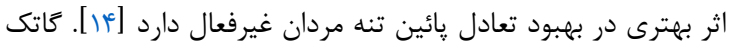

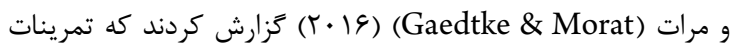

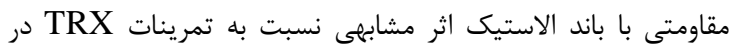

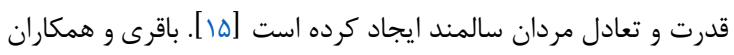


ارتفاع إ/ متر بالاتر از سطح سالن نصب شده بود، انجام گرديد. اين

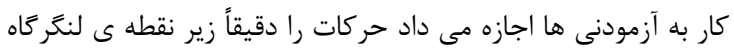

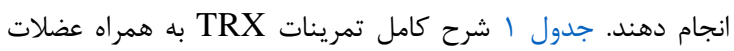

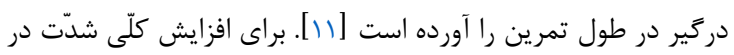
تمام تمرين هاى TRX در روند انجام تحقيق، اقداماتى از جمله تغيير

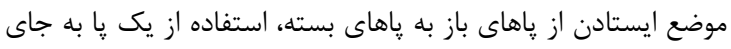

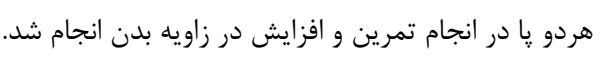

\section{تجزيه و تحليل داده ها}

در تجزيه و تحليل آمارى، ابتدا تمامى متغيرهاى كمّى توسط آزمون

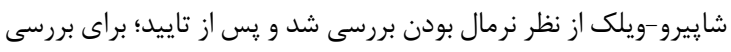

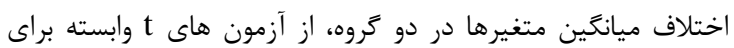

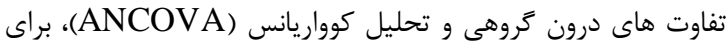
اندازه گيرى تفاوت هاى بين كروهى استفاده گَرديد. درصد تغييرات از

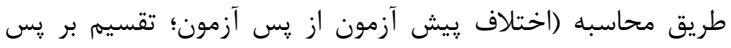

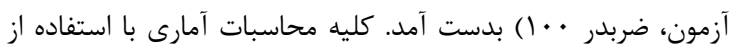

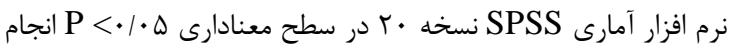

\section{يافتهها}

همانطور كه جدول r نشان مى دهد، بين متغيرهاى سن، وزن، قد و

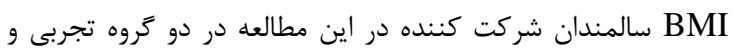

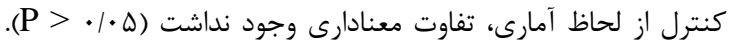
كروه تجربى با ميانگين (سن قد r.|• (سن س א نشان مى دهد، نتايج تحليل آمارى درون گروهى با استفاده از t وابسته

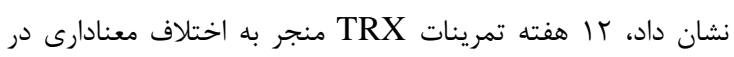

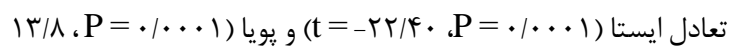

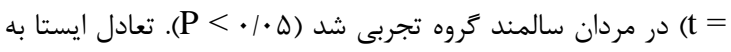

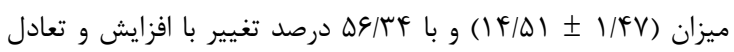

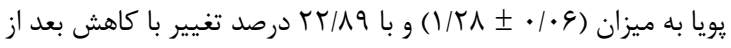
ז هفته تمرينات TRX همراه بود. از طرفى نتايج تحليل آمارى بين

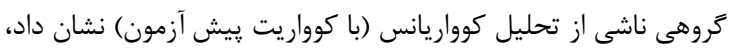

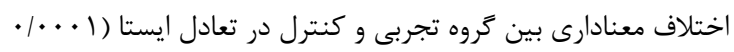

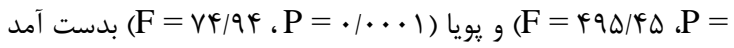

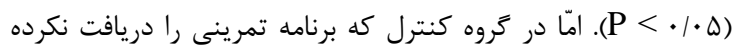

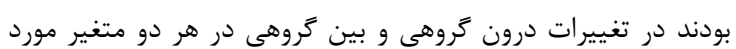

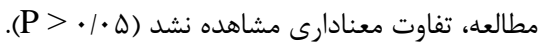

و مصرف هر نوع دارويى كه بر فاكتورهاى اندازه كيرى شده موثر باشد و وس) نداشتن حداقل دو جلسه فعاليت بدنى منظهم در هفته بود. بعد از

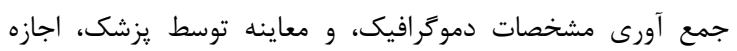
تمرينات ورزشى از طرف يزشك صادر شد. كليه شركت كنيند

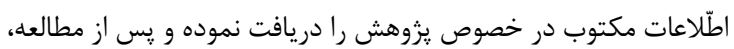
از آن ها درخواست شد، رضايت نامه كتبى را امضاء نمايند. در مرحله

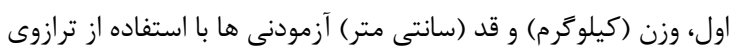
مدل SECA ساخت كشور آلمان، به ترتيب با دقت / / • كيلوگرم و

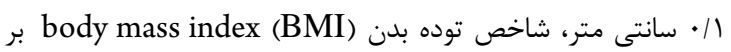
حسب وزن تقسيم بر مجذور قد (كيلوكرم بر مترمربع) اندازه كيرى شد. بدا.

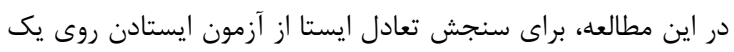

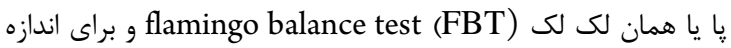

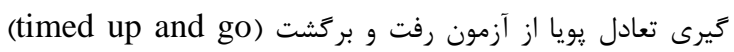

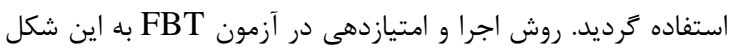

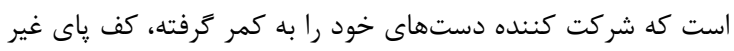

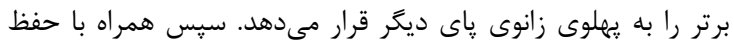

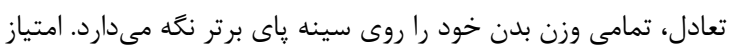

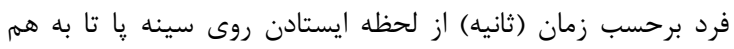

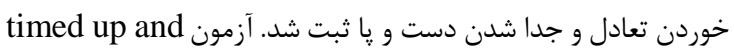

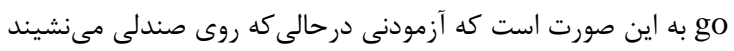

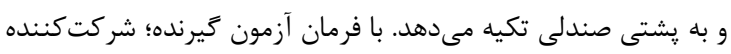
برمىخيزد و مسافت سه مترى علامت كذارى شده را مىي يُمايد. بعد از

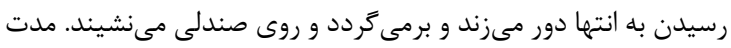

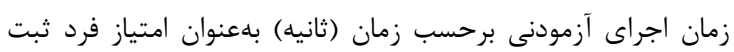

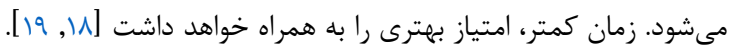
تعادل ايستا و يويا در پيش از تمرينات TRX و و در آخرين جلسه

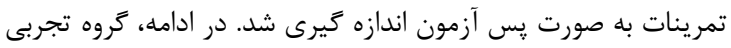

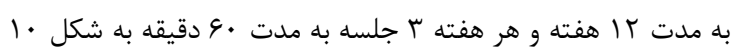

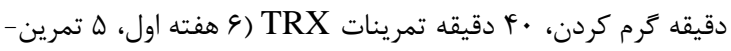

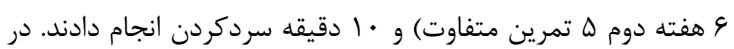

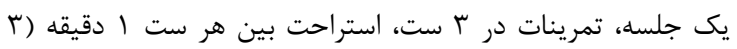

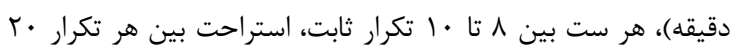

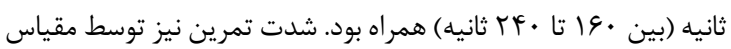

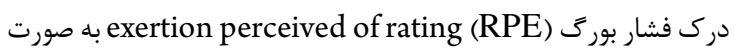
زير كنترل شد. بدين صورت كه قبل از شروع تمرينات، آزمودنى ها با بات بات

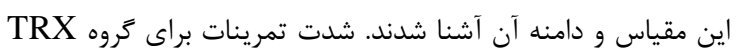

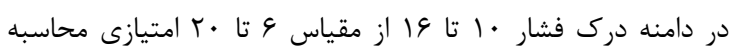

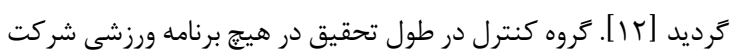

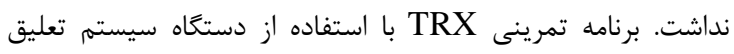

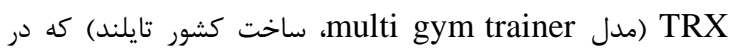


جدول ا. تمرينات TRX و نحوه ى صحيح انجام هر حركت و عضلاتى كه آن حركت را دركير مى كند [11].

\begin{tabular}{|c|c|c|}
\hline عضلات دركير شونده & توضيحات & تمرين TRX \\
\hline سينه اى بزرك، دلتوئيد، يشت بازو & 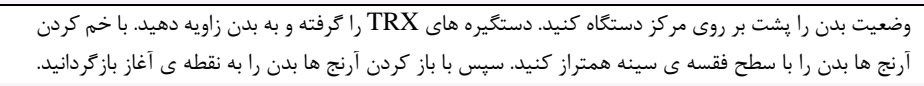 & يرس سينه \\
\hline سرينى سر ران، پشت ران (همسترينَ)، & 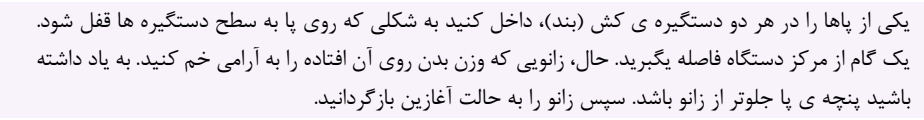 & لانث معلق(هر دو يا) \\
\hline (دوزنقه اى، عضله يشتى بزرگ، سرشانه & 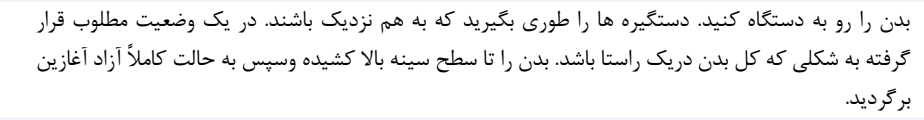 & يارويى(رويينگ) دو دست \\
\hline جهارسر ران، يشت ران، سرينى & 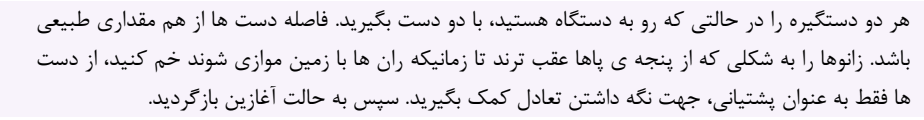 & 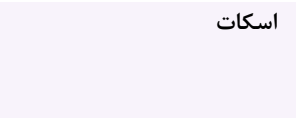 \\
\hline هاى لوزى شكل (دلتوييدى)، ذوزنقه اى، ماهيجه & 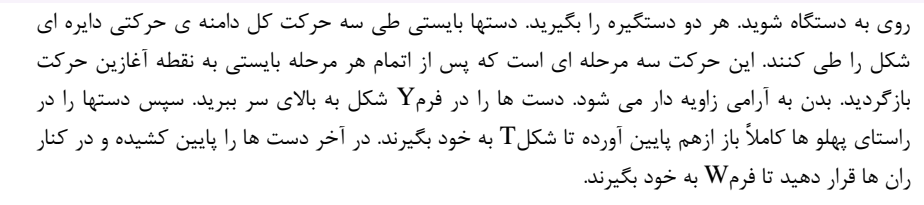 & 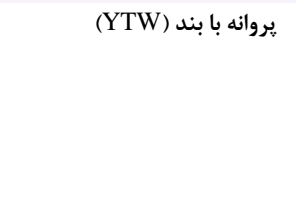 \\
\hline سرينى، يشت ران (همسترينَ) & 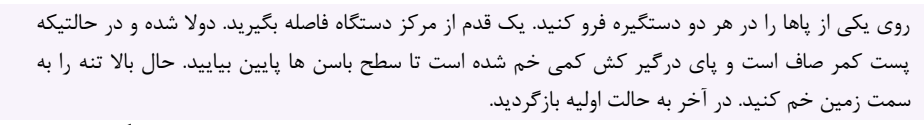 & يشت يا ددليفت (هر دو ها) \\
\hline يشت بازو & 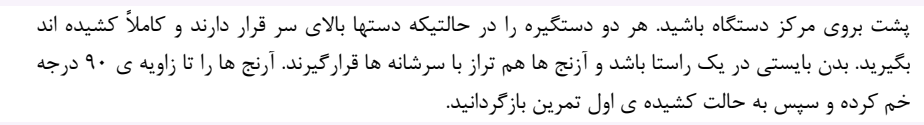 & يشت بازو \\
\hline يشت ران & 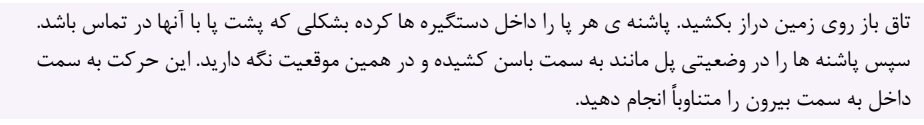 & 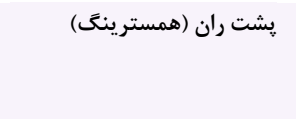 \\
\hline بهلو، فيله كمر راست شكم، عضله عرضى شكم، & 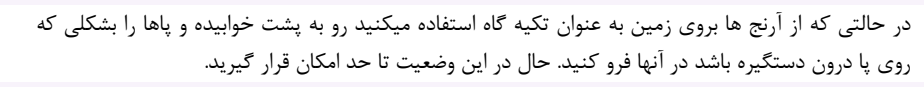 & پِلانك (تخت) \\
\hline يهلو، ماهيجه مربع كمرى & 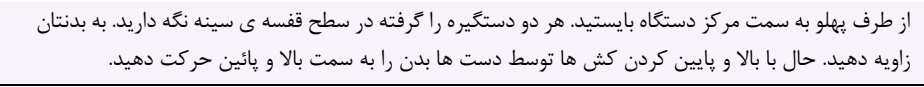 & $\begin{array}{r}\text { پالوف و بند (كش) سايد هولد با يرس } \\
\end{array}$ \\
\hline \multicolumn{3}{|c|}{ جدول r. ويثَى هاى توصيفى آزمودنى ها در كروه تجربى (n=1) و كنترل (n = (n) } \\
\hline $\mathbf{P}$ & ي پيش آزمون تجربى & متغير \\
\hline$\cdot 190$ & $91 / A V \pm 1 / T F$ & سن (سال) \\
\hline$\cdot / \pi \Delta$ & $V \Psi / r V \pm r / r V$ & وزن (كيلوكرم) \\
\hline$\cdot / \pi F$ & $1 / v r \pm \cdot / r q$ & قد (سانتى متر ) \\
\hline.$/ 1 \mathrm{~F}$ & TF/THE I/TG & BMI (كيلوكرم بر متر مربع) \\
\hline
\end{tabular}

جدول r. نتايج آزمون تحليل كوواريانس و t وابسته براى بررسى متغيرهاى يزوهش در كروه تجربى (n=1) و كنترل (n=1)

\begin{tabular}{|c|c|c|c|c|c|c|c|}
\hline \multicolumn{4}{|c|}{ ANCOVA } & \multicolumn{2}{|c|}{ درون تروهى t } & \multirow[t]{3}{*}{ انحراف معيار \ ميانكين } & \multirow[t]{3}{*}{ 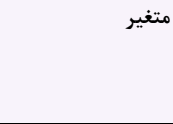 } \\
\hline \multicolumn{2}{|c|}{ كوواريت (ييش آزمون) } & \multicolumn{2}{|c|}{ متغير مستقل } & \multirow[t]{2}{*}{$\mathbf{P}$} & \multirow[t]{2}{*}{$\mathbf{t}$} & & \\
\hline $\mathbf{P}$ & $\mathbf{F}$ & $\mathbf{P}$ & $\mathbf{F}$ & & & & \\
\hline \multirow[t]{7}{*}{.$/ 94$} & $.1 \cdot 1$ & $\cdot(\cdots)^{* *}$ & $F q \Delta / \& \Delta$ & & & & تعادل ايستا (ثانيه) \\
\hline & & & & $\cdot|\cdots|^{*}$ & $-r t / k$. & & تجربى \\
\hline & & & & & & $\| / K F \pm \cdot / r$ & ي يش آزمون \\
\hline & & & & & & $r \Delta / V \Delta \pm I / V V$ & يس آزمون \\
\hline & & & & $\cdot / \Lambda$ & $\cdot \pi 4$ & & كنترل \\
\hline & & & & & & $11 / T 4 \pm \cdot / 19$ & ي پيش آزمون \\
\hline & & & & & & $\| 1 / T F \pm \cdot / 1 Q$ & يس آزمون \\
\hline \multirow[t]{7}{*}{$\cdot 119$} & $r q|\Delta|$ & $\cdot(\cdots)^{* *}$ & $V F / 9 F$ & & & & تعادل يويا (ثانيه) \\
\hline & & & & $\cdot|\cdots|^{*}$ & $1 r / 1$ & & تجربى \\
\hline & & & & & & G/AV $\pm \cdot / \Delta F$ & ي ريش آزمون \\
\hline & & & & & & $\Delta / \Delta q \pm \cdot / 4 \Lambda$ & يس آزمون \\
\hline & & & &.$/ 14$ & $-1 / 91$ & & كنترل \\
\hline & & & & & & V/FTE $\cdot 190$ & ي رِيش آزمون \\
\hline & & & & & & $V / F T \pm . / 94$ & يس آزمون \\
\hline
\end{tabular}


در اثر تمرين تعادلى برشمرد [بَ]]. بهبود تعادل در اثر تمرينات TRX

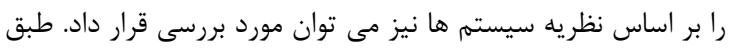

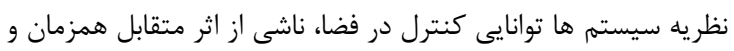

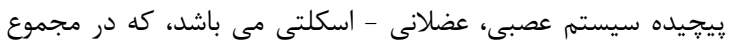

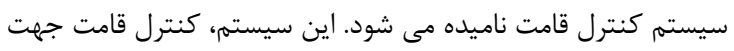

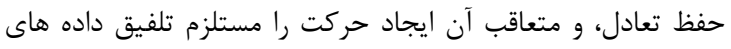
حسى، جهت تشخيص موقعيت بدن در فضا و همين طور توانايى

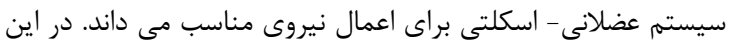

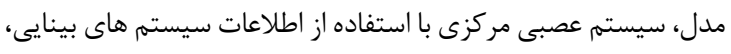
دهليزى و حس عمقى (شامل حس وضعيت مفاصل و حس محيطى)

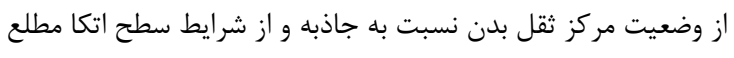

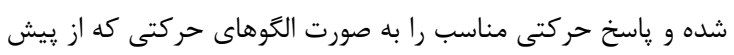

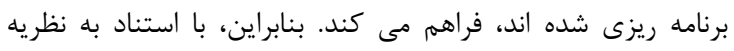

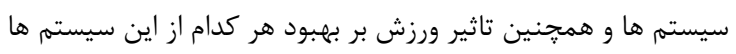

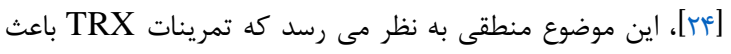

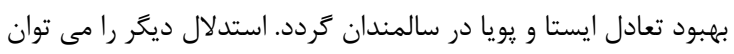
به دليل سازگًارى عصبى دانست. خرا كه تمرين بدنى موجب ساز

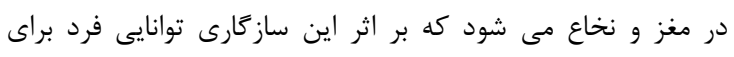

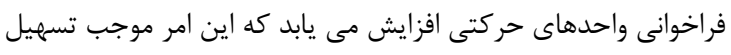

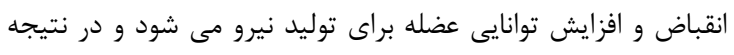

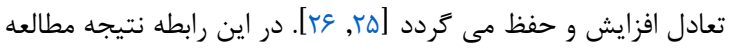

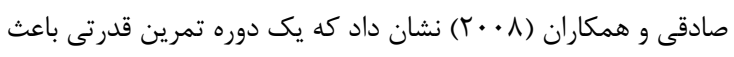

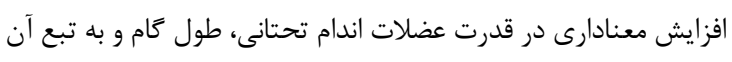

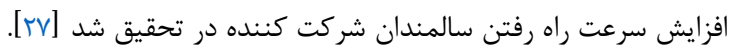

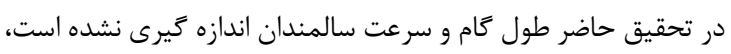
اما با توجه به افزايش قدرت عضلانى در اين تحقيق و نيز ارتباط بين قدرت عضلانى با طول كام و سرعت راه رفتن سالمندان شايد بتوان

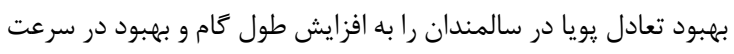

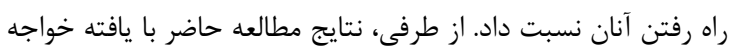

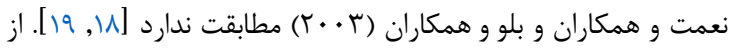

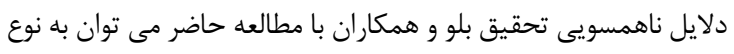

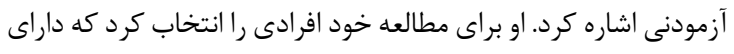

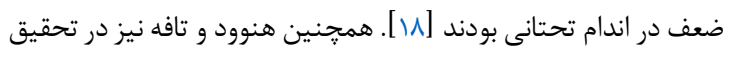

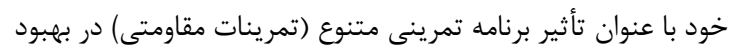
قدرت و عملكرد سالمندان نتوانست اثر فعاليت بدنى بر تعادل بويا و وني

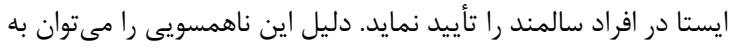

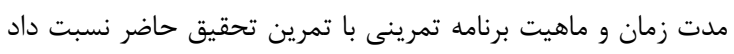

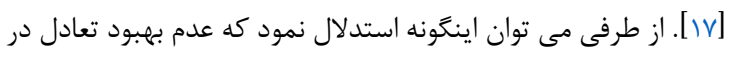

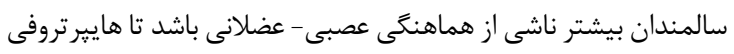

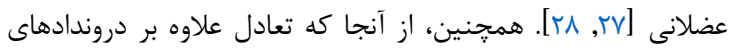

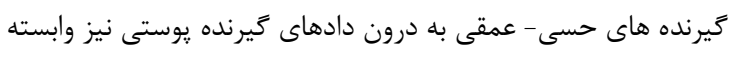

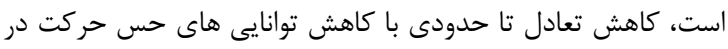

هدف از مطالعه حاضر، بررسى اثر I الهفته تمرينات TRX بر تعادل

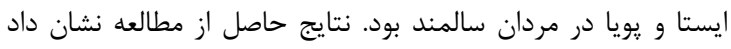

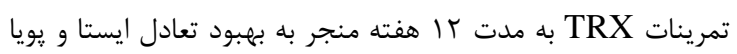

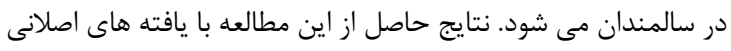

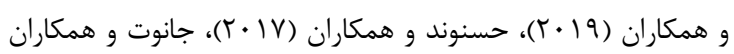
(Janot et al.)

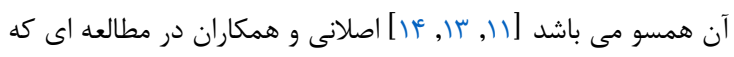
روى צץ مرد ميانسال انجام دادند، نتيجه كرفتند كه 1 هفته تمرينات

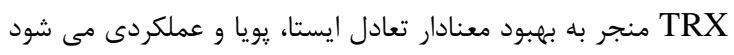

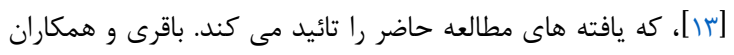

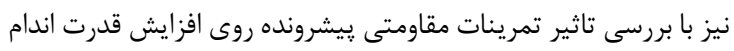

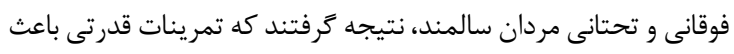

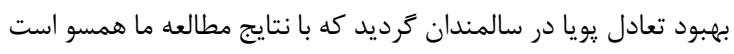

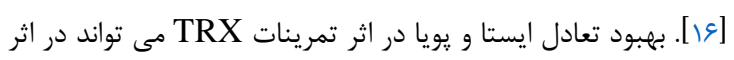
تقويت عضلات و بهبود قدرت عضلانى شركت كنندكان بدست آيد. جرا كه كاهش قدرت عضلانى اندام تحتانى، منجر به قرار كيرى مركز ثقل فئل

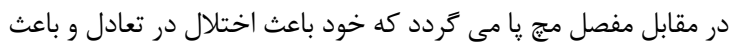

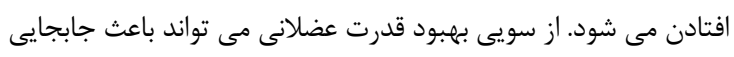

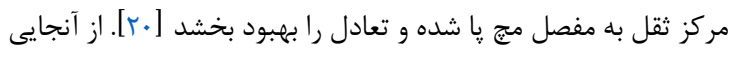

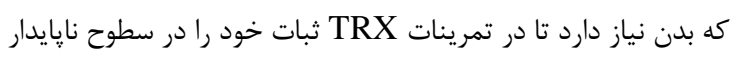
حفظ كند، اين مسئله به هماهنگَى سيستم عصبى كمك مى كن كند و

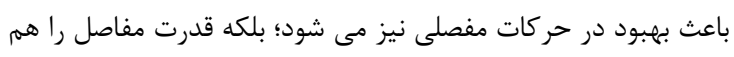

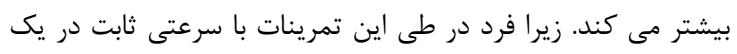

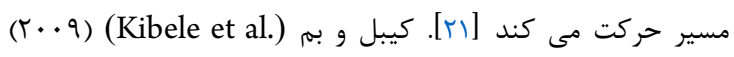
كزارش كردند كه تقويت عضلات ميان تنه با TRX و و وزن آنس آزاد، منجر

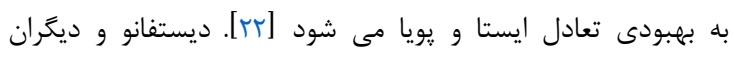
(r) (ristefano et al.)

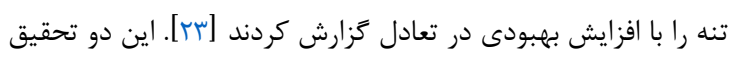

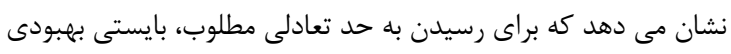

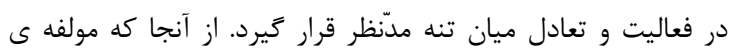

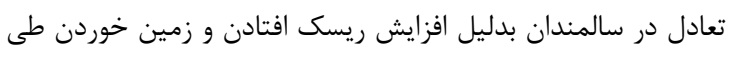

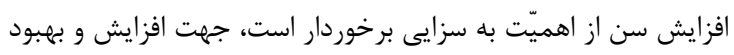

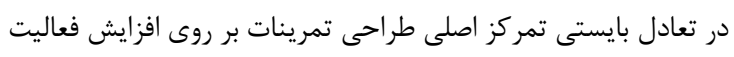

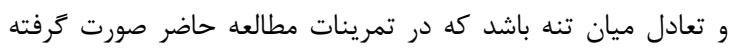

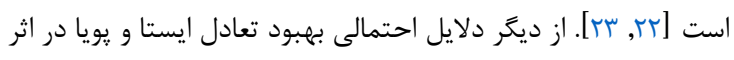

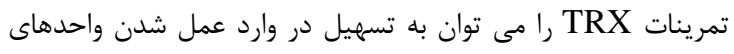

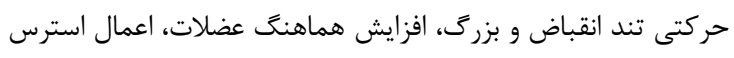

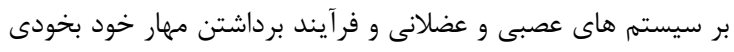

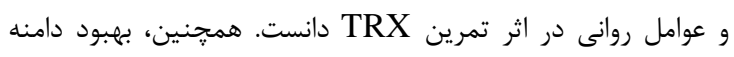
حركتى مفاصل، كنترل عصبى حركات و اعمال اضافه بار بر انتقال

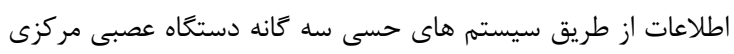


اطلاعات محفوظ خواهد ماند. همجنين تمامى شركت كنندكان در اين

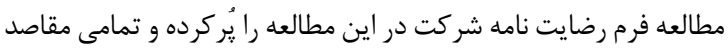
مطالعه براى شركت كننده گان به طور كامل شرح داده شده است.

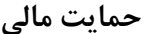

بخش از مطالعه حاضر تحت حمايت مالى معاونت محترم يزوهشى

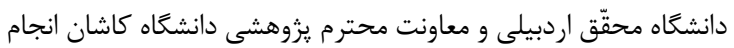

\section{سهم نويسندكًان}

آقاى محمدابراهيم بهرام ايده اصلى، نعارش اوليه، تهيه درافت اوليه

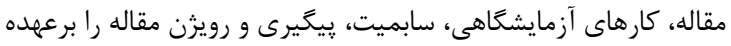

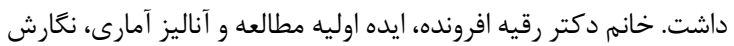
مقاله، رويزرن مقاله و نظارت روى يروزه را انجام داده و آقاى دكتر

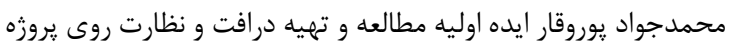
را به انجام رسانيد. تعارض منافع در اين مقاله هيج گَونه تعارض منافعى وجود ندارد. كاربرد عملى مقاله اين مطالعه براى بهبود تعادل در سالمندان و كيفيت زندكَى و سلامت روان آنها مفيد مى باشد.

سياسگذارى محققين اين يزوهش، از كليه آزمودنى هايى كه در اين يزوهش شركت

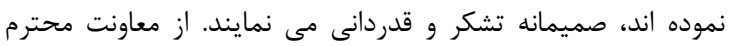

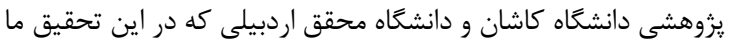
را همر اهى نمودند، سياسگزاريم.

\section{References}

1. Bize R, Johnson JA, Plotnikoff RC. Physical activity level and health-related quality of life in the general adult population: a systematic review. Prev Med. 2007;45(6):401-15. doi: 10.1016/j.ypmed.2007.07.017 pmid: 17707498

2. Benjuya N, Melzer I, Kaplanski J. Aging-induced shifts from a reliance on sensory input to muscle cocontraction during balanced standing. J Gerontol A Biol Sci Med Sci. 2004;59(2):166-71. doi: 10.1093/gerona/59.2.m166 pmid: 14999032

3. De Bruin ED, Murer K. Effect of additional functional exercises on balance in elderly people. Clin Rehabil. 2007;21(2):112-21. doi: 10.1177/0269215506070144 pmid: 17264105

4. Wojtek J, David N, Maria A, Christopher T, Claudio R, George J, et al. American College of Sports Medicine position stand. Exercise and physical activity for older adults. J Am Coll Sports Med. 2009;41(7):1510-30. doi: 10.1249/MSS.0b013e3181a0c95c pmid: 19516148

5. Butler AA, Lord SR, Rogers MW, Fitzpatrick RC. Muscle weakness impairs the proprioceptive control of human
افراد مسن مرتبط است. همجنين، كاهش حداكثر قدرت عضلات نيز

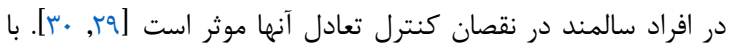

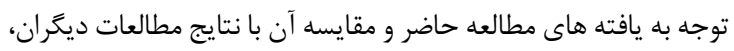

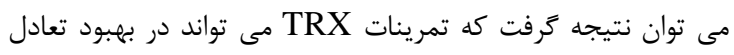

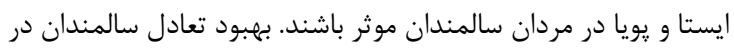

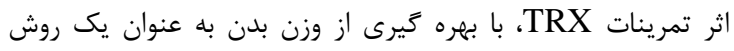

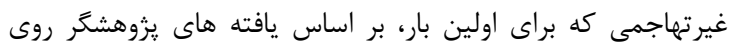

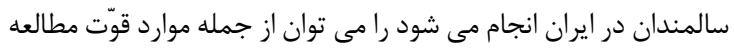

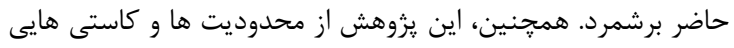
برخوردار بود، كه مى توان به انتخاب حجم نمونه، عدم كنترل كامل

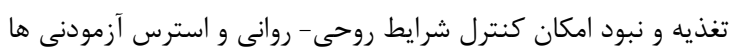
در طول اجراى يزوهش اشاره نمود. نتيجه كيرى

يافته ها نشان مى دهد كه ورزش TRX مى تواند يك ابزار مفيد براى

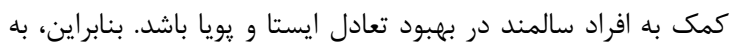
نظر مى رسد بتوان به كمك اين ورزش، يِامد هاى منفى جسمانى و

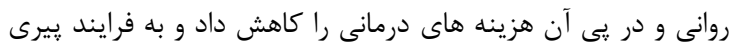

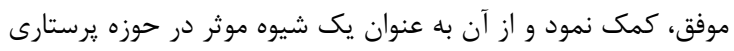
و توانبخشى سالمندى مورد استفاده قرار داد.

ملاحظات اخلاقى اين كارآزمايى بالينى در داملى سانه IRCT به شماره

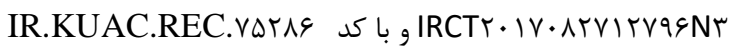
به ثبت رسيد. نويسندگان اين مقاله طبق يروتكل هاى اخلاقى مطالعات

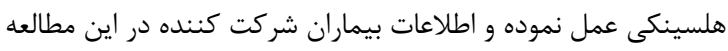

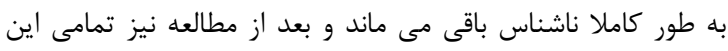

standing. Brain Res. 2008;1242:244-51. doi: 10.1016/j.brainres.2008.03.094 pmid: 18499088

6. Chiacchiero M, Dresely B, Silva U, DelosReyes R, Vorik $B$. The relationship between range of movement, flexibility and balance in the elderly. Top Geriatr Rehabil. 2010;26(2):148-55.

doi: 10.1097/TGR.0b013e3181e854bc

7. Taghadosi M, Mirbagher N, Torabian M, Sedaghati P. Investigating the effect of Pilates exercises on anxiety in women with diabetes type II. Cmja. 2014;4(1):687-99.

8. Bahram M, Pourvaghar M, Akkasheh G. The effect of 8 weeks pilates training on depression treatment on retired work men. Jgn. 2015;1(2):31-42.

9. McGill SM, Cannon J, Andersen JT. Analysis of pushing exercises: muscle activity and spine load while contrasting techniques on stable surfaces with a labile suspension strap training system. J Strength Cond Res. 2014;28(1):105-16. doi: 10.1519/JSC.0b013e3182a99459 pmid: 24088865

10. Snarr RL, Esco MR. Electromyographic comparison of traditional and suspension push-ups. J Hum Kinet. 
2013;39:75-83. doi: 10.2478/hukin-2013-0070 pmid: 24511343

11. Janot J, Heltne T, Welles Chelsea RJ, Anderson H, Howard A, Lynn MS. Effects of TRX versus traditional resistance training programs on measures of muscular performance in adults. J Sports Med. 2013;2(2):23-38.

12. Whitehurst MA, Johnson BL, Parker CM, Brown LE, Ford AM. The benefits of a functional exercise circuit for older adults. J Strength Cond Res. 2005;19(3):647-51. doi: 10.1519/R-14964.1 pmid: 16095420

13. Aslani M, Kalantariyan M, Minoonejad H. Effect of functional training with TRX on balance of middle-aged men. J Rehab Med. 2019;7(4):80-9.

14. Hasanvand H, Ranjbar R, Habibi AH, Goharpey Sh. Comparison of the effect of TRX and traditional resistance training on some factors of body composition andbalance in sedentary men. Jundishapur Sci Med J. 2017;16(6):621-30.

15. Gaedtke A, Morat T. Effects of two 12-week strengthening programmes on functional mobility, strength and balance of older adults: Comparison between TRX suspension training versus an elastic band resistance training. Cejssm. 2016;13(1):49-64. doi: 10.18276/cej.2016.1-05

16. Bagheri H, Abdolvahab M, Raji P, Jalili M, Faghih Zadeh $S$, Soltani $Z$. The effects of progressive resistive exercises on activities of daily living of elderly persons. Mrj. 2010;4(1-2):56-9.

17. Henwood TR, Taaffe DR. Short-term resistance training and the older adult: the effect of varied programmes for the enhancement of muscle strength and functional performance. Clin Physiol Funct Imagin. 2006;26(5):305-13. doi: 10.1111/j.1475097X.2006.00695.x pmid: 16939509

18. Bellew JW, Yates JW, Gater DR. The initial effects of lowvolume strength training on balance in untrained older men and women. J Strength Cond Res. 2003;17(1):121$8 . \quad$ doi: $10.1519 / 1533$ 4287(2003)017<0121:tieolv>2.0.co;2 pmid: 12580667

19. Khaje-Nemat K, Sadeghi H, Sahebozamani M. The effect of 8 weeks of strength training on static and dynamic balance in healthy men. Sport Med. 2014;6(1):45-55.

20. Kaesler DS, Mellifont RB, Kelly PS, Taaffe DR. A novel balance exercise program for postural stability in older adults: a pilot study. J Bodywork Mov Therap. 2007;11(1):37-43. doi: 10.1016/j.jbmt.2006.05.003

21. Irez GB. The effects of different exercises on balance, fear and risk of falling among adults aged 65 and over.
Anthropologist. 2014;18(1):129-34. doi: 10.1080/09720073.2014.11891528

22. Kibele A, Behm DG. Seven weeks of instability and traditional resistance training effects on strength, balance and functional performance. J Strength Cond Res. 2009;23(9):2443-50. doi: 10.1519/JSC.0b013e3181bf0489 pmid: 19952576

23. Distefano LJ, Distefano MJ, Frank BS, Clark MA, Padua DA. Comparison of integrated and isolated training on performance measures and neuromuscular control. J Strength Cond Res. 2013;27(4):1083-90. doi: 10.1519/JSC.0b013e318280d40b pmid: 23364296

24. Critchley DJ, Pierson Z, Battersby G. Effect of pilates mat exercises and conventional exercise programmes on transversus abdominis and obliquus internus abdominis activity: pilot randomised trial. Man Ther. 2011;16(2):183-9. doi: 10.1016/j.math.2010.10.007 pmid: 21075038

25. Nagy E, Feher-Kiss A, Barnai M, Domjan-Preszner A, Angyan L, Horvath G. Postural control in elderly subjects participating in balance training. Eur J Appl Physiol. 2007;100(1):97-104. doi: 10.1007/s00421-007-0407-x pmid: 17333243

26. Donyapour H, Mohammadzade H, Abedini M, Rezaye S, Safari H. The impacts of Pilates trainings on improvements of dynamic balance and gait performance in elderly men with falling background. J Rehab Med. 2013;2(3):11-8.

27. Sadeghi H, Norouzi H, KarimiAsl A, Montazer M. Functional training program effect on static and dynamic balance in male able-bodied elderly. Salmand. 2008;3(2):565-71.

28. Surbala L, Khuman PR, Trivedi P, Devanshi B, Mital V. Pilates versus conventional balance training on functional balance and quality of life in elderly individuals: A randomized controlled study. Sch J App Med Sci. 2014;2(1):221-6.

29. Khoshmaram F, Sadeghi H, Eftekhari F. Electromyographic activity of selected core muscles during suspension workouts. J Rehab Med. 2019;8(3):140-9. doi: 10.22037/jrm.2019.111184.1821

30. Appell IPC, Pérez VR, Nascimento MDM, Coriolano HJA. The Pilates method to improve body balance in the elderly. Arch Exercise Health Disease. 2012;3(3):188-93. doi: 10.5628 /aehd.v3.i3.126 